http://jmscr.igmpublication.org/home/

ISSN (e)-2347-176x ISSN (p) 2455-0450

crossref DOI: https://dx.doi.org/10.18535/jmscr/v8i6.75

Journal Of Medical Science And Clinical Research

\title{
Efficacy of threshold inspiratory muscle training on improving endurance capacity and quality of life in mitral valve replacement
}

\author{
Authors \\ Dr V. Kiran M.P.T Professor in Physiotherapy, R. Venkatesan Asst Professor, \\ TNPSU, G. Nusarth Jaha MPT Asst Professor, C. Varalakshmi MPT, Asst Professor \\ J.Anandh Raj MPT, Asst Professor
}

\begin{abstract}
Background: Mitral valve replacement was done in $80 \%$ of cases with valvular heart diseases (Gummer et al., 2008). Despite advances in reparative techniques, valve replacement surgery is considered as treatment option to restore proper heart function in people with mitral valve disease (Alain Pratt et al., 2001). Mitral valve replacement were accomplished in 1960 by harken and Starr. Diseases of the heart valves constitute a major cause of cardiovascular morbidity and mortality worldwide with an enormous burden on healthcare resources.

Specific Objective: To compare the effect of Threshold inspiratory muscle training device along with Conventional physiotherapy on the endurance capacity and quality of life in mitral valve replacement.

Design: Pre-test and Post-test experimental study design

Study Settings: Department of Physiotherapy, Narayana hospital, Nellore.

Intervention: 30 patients were selected and divided in to two groups Group $-A$ was given with conventional physiotherapy and Group $-B$ was given with Threshold inspiratory muscle training .and total duration was 8 weeks and 3 session per week and $30-40$ min per session.

Out cum measures: six minute walk test, short form - 36 questionnaire

Result \& Conclusion: The purpose of the study is to find out the effect of analyze the efficacy of Threshold Inspiratory Muscle Training Device Compare with Conventional Breathing Exercises on Improving endurance capacity and Quality of Life in Mitral Valve Replacement. Based on this statistical analysis the Group B participants show a marked improvement in the six minute walk test and the quality of life questionnaire when compared to Group A participants. Therefore, this study strongly suggests that inspiratory muscle training when added to conventional physiotherapy program proved to be significantly effective in improving endurance capacity and quality of life as compared to conventional physiotherapy alone in mitral valve replacement
\end{abstract}

Keywords: MVR, Threshhold inspiratory muscle training.

\section{Introduction}

Diseases of the heart valves constitute a major cause of cardiovascular morbidity and mortality worldwide with an enormous burden on healthcare resource is a significant health problem all over the world, although it is far less common than coronary artery disease. It is characterized by impaired function of one or more of the four cardiac valves. Heart valves may go for any of the two functional abnormalities like stenosis or regurgitation. When both stenosis and regurgitation are present in the same valve, one process usually predominates

India contributes to nearly $25 \%$ to $50 \%$ of the global burden of valvular heart disease, with $47.2 \%$ in mitral stenosis, $24 \%$ as mitral regurgitation, around $11.2 \%$ in aortic regurgitation and aortic stenosis with $18.0 \%$. Mitral valve problem is one of the common valve problems (Padmavathi S 2001). Among the patients with 
isolated mitral valve (MV) involvement, pure mitral stenosis was the dominant lesion (37.1\%). Another $35.7 \%$ of cases had mitral regurgitation in addition to mitral stenosis; thus, mitral stenosis was seen in a total of $72.8 \%$ of patients. Incidences of mitral regurgitation were higher in men but mitral stenosis was higher in women Mitral stenosis was first reported in 1668 by John Mayow, and until recently was the most discussed valvular heart disease. The incidence of valve related events was significantly higher in group of patients with age 25-45years (Ambler et al., 2005). A variety of etiologies can lead to valvular heart disease, including myxomatous degeneration, congenital malformation, infective endocarditis, rheumatic heart disease, and other acquired disorders. Valvular disorders more commonly affect the aortic and mitral valves than those on the right (tricuspid and pulmonic) (Bender et al., 2002).

Mitral valve stenosis possess smaller valve opening than normal due to stiff or fused leaflets. As stenosis severity worsens, blood flow restriction limits left ventricular output. As the left atrial pressure rises to compensate for mitral valve narrowing, a mean trans-vascular pressure gradient of 10 to $15 \mathrm{mmHg}$ may occur at rest; left atrial pressure and gradient rise substantially with exercise (Fann et al., 1997). Finally it increases work load to heart

Clinical signs of mitral regurgitation include a systolic heart murmur with an apical or axillar point of maximum, weakness, fatigue, Dyspnea and palpitation. Fatigue and exercise intolerance typically cause patients with mitral stenosis to adopt a gradually more sedentary lifestyle (Poyyamozhi et al., 2016). The end- stage of this development is heart failure, and in advanced cases, pulmonary congestion and even of right side heart failure (Thierry Le Tourneau et al., 2000). The degree of heart failure is described by the New York Heart Association (NYHA)-class's I-IV. Surgery remains the therapeutic option related to better survival of individuals with valvular heart disease. Heart valve surgery is the second-most- common type of cardiac surgery, accounting for $20 \%$ to $35 \%$ of all cardiac surgical procedures, with an in-hospital mortality of $4 \%$ to 8\%. (Geissler et al., 2009) declared in their study that 30,000 heart valve replacement surgeries are performed annually due to increase prevalence of degenerative valve diseases in developed countries. The mentioned result may be due to more mitral valve replacement requirement in younger adults that essentially accompanies more complications compared with aortic and pulmonary valve replacement. The decision regarding the type of corrective surgery was made by the cardiovascular surgeon on the basis of preoperative data and after assessment of the anatomic status of the mitral valve during surgery. Most valve replacement operations are performed through a median sternotomy incision, this procedure may alter the ribcage biomechanics due to its extensive handling and prolonged duration of surgery (Williams et al., 2008). This results in altered pulmonary mechanics by decreasing lung compliance, increasing airway resistance and work of breathing because the chest wall no longer impedes lung expansion (Gravlee et al., 2007 \& Passaroni et al., 2015). Most valve replacement operations are performed through a median sternotomy incision, this procedure may alter the ribcage biomechanics due to its extensive handling and prolonged duration of surgery (Williams et al., 2008). This results in altered pulmonary mechanics by decreasing lung compliance, increasing airway resistance and work of breathing because the chest wall no longer impedes lung expansion.

The threshold loading device has an advantage of being independent of inspiratory flow rate but requires a build-up of negative pressure before flow is initiated.

Inspiratory muscle training with specially designed device called threshold breathing device, which can adjust to produce reliable Inspiratory pressure loads that were independent of airflow. With this device, the Inspiratory pressure load varies according to the size of the Inspiratory orifice and rate of Inspiratory airflow 


\section{Need for the Study}

Valvular heart disease (VHD) is a significant health problem all over the world, although it is far less common than coronary artery disease. Valvular disorders more commonly affect the aortic and mitral valves (Bender et al., 2002) than those on the right (tricuspid and pulmonic). Mitral valve disease is most prevalent.The end-stage of this disease is the development is heart failure, and in advanced cases, pulmonary congestion and even of right side heart failure (ThierryLe Tourneau et al., 2000). Surgical treatment remains the therapeutic option related to better survival of individuals with valvular heart disease.

Hence, assessment of lung function becomes an important factor post- operatively. Also the intervention needs to be targeted according to the postoperative complications like airway clearance techniques or strengthening the respiratory muscles, etc. The evidence suggests that some interventions recently used in physiotherapy may be no benefit to patients undergoing uncomplicated open heart surgery (Patman S, 2001).Apart from conventional physiotherapy treatment, Aiming to re-establish endurance capacity and quality of life, some strategies should be used, such as inspiratory muscle training, which improves endurance capacity .A few studies were done on graded threshold inspiratory muscle training protocol for mitral valve replacement. So, more investigations are needed to know its effect in regular care of patients with mitral valve replacement, therefore the need for this study and the study was undertaken.

\section{Aim of the Study}

To investigate the efficacy of Threshold inspiratory muscle training device on the endurance capacity and quality of life in mitral valvereplacement

\section{Objectives of the Study}

- To study the effect of Conventional physiotherapy on the endurance capacity and quality of life in mitral valve replacement.
- To study the effect of Threshold inspiratory muscle training device on the endurance capacity and quality of life in mitral valve replacement.

- To compare the effect of Threshold inspiratory muscle training device along with Conventional physiotherapy on the endurance capacity and quality of life in mitral valve replacement.

\section{Hypothesis}

Null Hypothesis: There is no significant difference in effectiveness of Threshold inspiratory muscle training device on improving the endurance capacity and quality of life in mitral valve replacement.

Alternative Hypothesis: There is a significant difference in effectiveness of Threshold inspiratory muscle training device on improving the endurance capacity and quality of life in mitral valve replacement.

\section{Methodology}

Study Design: Pre-test and Post-test experimental study design.

Study Setting: The study was conducted in the Department of Cardio-Thoracic Surgery and Department of Physiotherapy, Narayana hospital, Nellore.

Study Duration: The study was conducted over a period of one year. Each patient underwent treatment for the duration of 8 weeks. Frequency is three sessions per week Duration, \& 30-40 minutes per session

Study Samples: A total of 30 patients were selected based on the selection criteria and divided into two groups. Each group consisted of $(n=15)$ members. The sample selection was done using random sampling method. The informed written consent was obtained from the subjects after explaining the purpose and procedure of the study in the language they understood best.

Inclusion Criteria: Both genders, Age of 30-40 years (low postoperative Inspiratory Capacity), Only Mitral valve replaced patients are included, 
Patients with LVEF $>45 \%$, Subjects willing to participate in the study, Subject who can understand the language better

Exclusion Criteria: Prolonged ICU (>48hrs), ICD tube $(>24 \mathrm{hrs})$, ventilators $(>12 \mathrm{hrs})$, Who did not understand/refused to do the technique, Patient who are not willing to buy device, Complicated and readmitted to ICU, Congenital mitral valve disease / Double valve replacement, Cardiomyopathy patients, Left ventricular dysfunction/ Pulmonary hypertension

Variables Independent Variables: Threshold Inspiratory Muscle Training, Conventional physiotherapy

Dependent Variables: Endurance Capacity, Quality of Life

Operational Tools: Six Minute Walk Test, Short Form - 36 Questionnaire

Procedure: Post-surgery mitral valve replacement patient has been shifted from cardiac ICU to cardiac inpatient unit on third postoperative day. All the patients were assessed by cardiothoracic surgeons and stated to be haemodynamically stable and recommended for cardiac rehabilitation. Based on the inclusion criteria and willingness of patients, the subjects were recruited for the study, around of 60 surgeries were done in the period of six months. The participants were recruited with the prescription from the surgeon and a clear assessment was done to find the suitable participants in this study. The patients were divided into 2 groups by Lottery method, each group consists of 15 patients with postoperative mitral valve replacement.

A clear instruction was given to all the patients who signed up for the study, written consent was obtained from every individual patient. Instruction about the exercises, beneficial effect of the exercises, demerits of exercises were told to the patients, log book use was instructed to the patient and invited them to visit the OPD physiotherapy department during their next visit with the doctor. 6 minute walk test was instructed and asked them to perform in the hospital corridor with the markings over the floor and SF36 questionnaire was given to them asked to fill-up the questionnaire, some assistance was provided by the team of physiotherapy during the questionnaire fill-up. The collected data were kept confidentially and taken for the analysis.

Group-A: Patients in this group underwent Conventional physiotherapy as the exercises and the protocols elaborated in the appendix. Duration -30 minutes/ session, Frequency -Twice a day (3 days/ week for 8 weeks).Conventional physiotherapy received diaphragmatic breathing exercise, upper and lower limb exercises, stair climbing activities

Group-B: Patients in this group underwent Threshold inspiratory muscle training used in Hulzebos study along with Conventional physiotherapy. The inspiratory threshold loading device (Philips Respironics) was used. Inspiratory muscle training was commenced on the $3^{\text {rd }}$ day; three sessions a day initially to familiarity with device. Three sets of ten inspiratory maneuvers were conducted in one session with a rest period of one minute between the sets.

The patients were asked to breathe in through the mouthpiece with the nose clip placed in situ, so that all breathing was done through the mouth. Instructions were given to breathe in deep but slow for the full duration of inspiration as trained earlier. The resistant ports were changed according to the subject's capacity while the patient was instructed to feel a moderate amount of resistance while breathing in and without causing any disturbances in respiratory pattern.

Patients were asked to record day in and day out activities and limitations faced by them in a $\log$ book. The researcher reviewed the logbook after 8 weeks period when patient visited the Cardio thoracic outpatient for follow up, the exercises and the protocols elaborated in the appendix.Duration40-45 minutes/ session with Frequency Twice a day (3 days/ week for 8 weeks). 


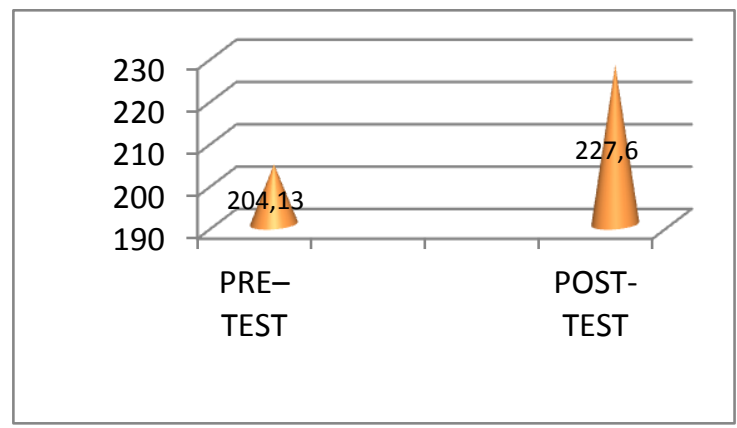

Group -A 6 min walk test

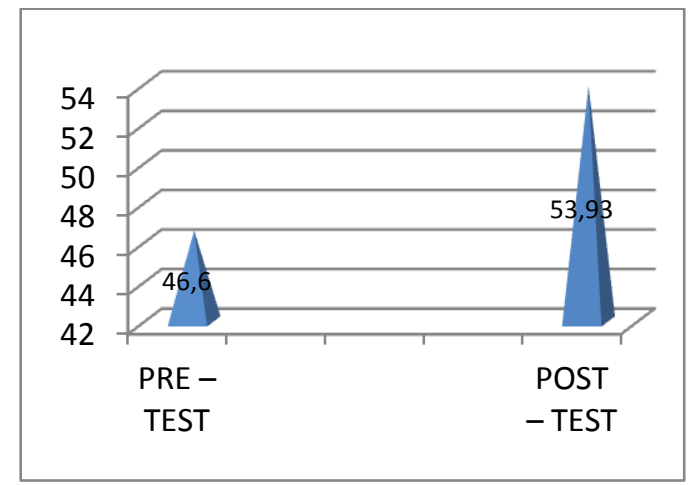

Group -A short form - 36 questionnaires

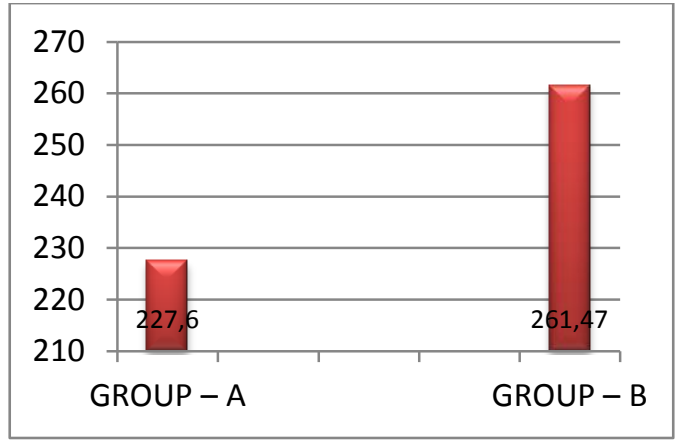

Group A \& B 6 min walk test

30 subjects with postoperative Mitral valve replacement were selected according to inclusive and exclusive criteria and randomly divided in to two groups with 15 subjects in each experimental group (Group A and Group B). Age group of the participants varies from 30 years to 40 years. Treatment duration was 8 weeks.

The Paired ' $t$ ' test analyses for the pre test and post test variable of six minute walk test for the Group A and Group B patients with mitral valve replacement which was shown in table I and II. Both the groups show significant differences in the pre test and post test values. The ' $t$ ' value for the Group A is 19.84 and the't' value for the Group B is 35.11. The unpaired' test analysis for the post-test variables of both group for the six minute walk test for measuring endurance capacity

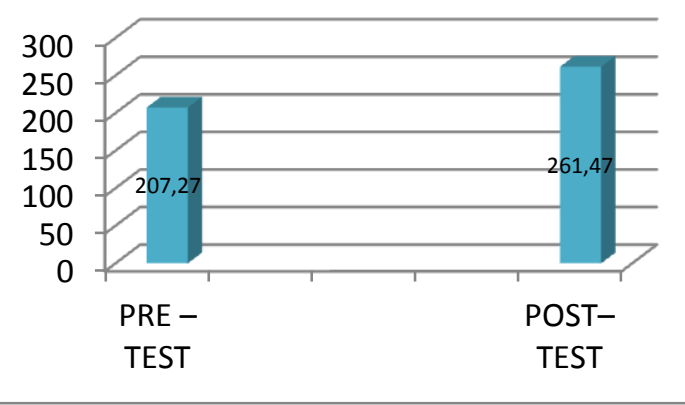

Group-B 6 Min walk test

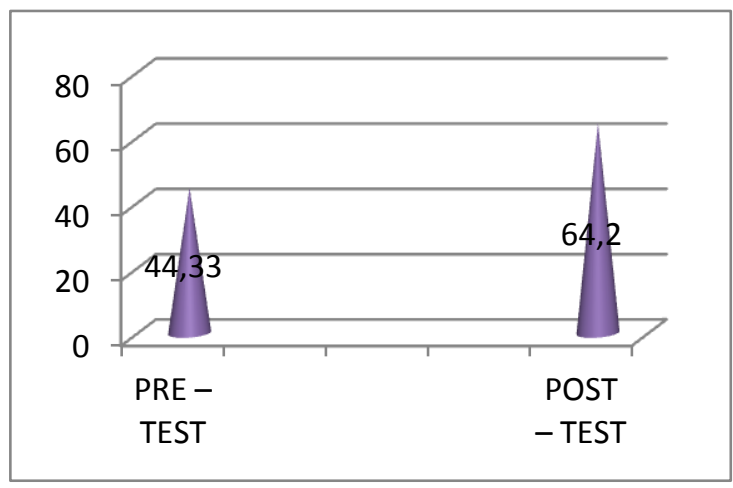

Group -B short form - 36 questionnaires

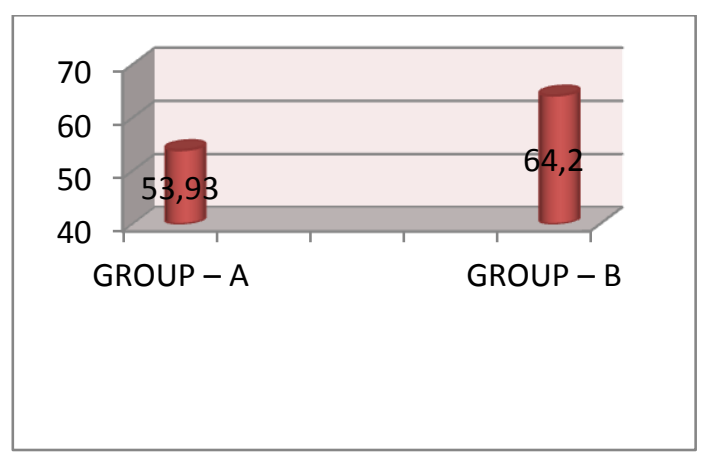

Group A \& B Short form 36 questioners

in patients is shown in the table III. There was a significant difference shown between the Groups. Subjects in Group A showed fewer improvements as that of Group B. The unpaired ' $t$ ' value for the post test variables for both groups is 21.53 .

The Paired ' $t$ ' test analyses for the pre test and post test variable for the six minute walk test for measuring endurance capacity in patients with mitral valve replacement which was shown in table IV and V. Both the groups show significant The purpose of the study is to find out the effect of analyze the efficacy of Threshold Inspiratory Muscle Training Device Compare with Conventional Breathing Exercises on Improving endurance capacity and Quality of Life in Mitral Valve Replacement.

30 patients with postoperative mitral valve 
replacement were selected randomly for the study. All the participants were assessed for hemodynamic stability by a cardiac surgeon and were assessed by the clinical physiotherapist prior to the recruitment. All the participants were recruited based on the selection criteria. A clear explanation was given to every individual subjects and those who were selected and randomly assigned into two equal groups. Written consent was obtained from all the participants.

Discussion:-Group A (control group) participants underwent conventional physiotherapy based on postoperative breathing exercise protocol used on follow up period, for duration of 8 weeks and to maintain mobility. Group B (experimental group) was given Threshold Inspiratory Muscle Trainer (Threshold IMT device HS730) the training parameters are based on a set of treatment protocol. Following the treatment, their pre test values and the post test values were calculated and analyzed for the results. The results were analyzed using tools of measurement of

Six Minute Walk Test and Short Form 36 Questionnaire. The data was collected, the results were tabulated and the outcome was analyzed using paired and unpaired test.

Student ' $t$ ' test was used to find the difference between the pre-test and post - test outcomes as well as the difference between the two groups. Results of Group - A and Group - B, post-test values showed that calculated ' $\mathrm{t}$ ' value is greater than critical value using Six Minute Walk Test and the Quality of life.

Based on this statistical analysis the Group B participants show a marked improvement in the six minute walk test and the quality of life questionnaire when compared to Group A participants.

Therefore, this study strongly suggests that inspiratory muscle training when added to conventional physiotherapy program proved to be significantly effective in improving endurance capacity and quality of life as compared to conventional physiotherapy alone in mitral valve replacement.

\section{Conclusion}

There is a significant improvement of 6 minute walk test. in both the groups. There is a significant improvement of Quality of life in both the groups. When compared to Group A (Control group), the Group B (Experimental group) shows a marked improvement of 6 minute walk test. When compared to Group A (Control group), the Group B (Experimental group) shows a marked improvement of Quality of life. So this study concluded that inspiratory muscle training when added to conventional physiotherapy program proved to be significantly effective in improving endurance capacity and quality of life as compared to conventional physiotherapy alone in mitral valve replacement.

\section{Bibliography}

1. Akansha Dixit, Shashwat Prakash. Effects of threshold inspiratory muscle training versus conventional physiotherapy on the weaning period of mechanically ventilated patients: a comparative study. Int J Physiother Res 2014;2(2):424-28.

2. Akhtar R, Jalwan J, Mukherjee S, To Compare the Immediate Effect of Deep Breathing Exercise and Incentive Spirometry on ABG after Mitral Valve Replacement Surgery. IJOPT; 2012; Vol 6, No 4; 12-17

3. Alain Pratt: Rest and exercise in the treatment of heart disease. South Med J, pp. 481-485

4. Alfredo josé rodrigues Paulo eduardo gomes ferreira, paulo roberto barboza évora: Effects of an Inspiratory Muscle Rehabilitation Program in the Postoperative Period of Cardiac Surgery, (Arq Bras Cardiol 2009;92(4):261- 268).

5. Ambler G, Omer RZ, Royston P, Kinsman R, Keogh BE, Taylor KM: Generic, simple risk stratification model for heart valve surgery. Circulation. 2005 jul 12; 112(2); 224-31. Epub 2005 jul 5.

6. American Heart Association: measuring and improving quality of care, ( 
CIR.101:1483-1493.12.1483)

7. Andell P, Martinsson A, Andersson C, Stagmo M: epidemiology of valvular heart disease in a Swedish nationwide hospital based register study. Heart. 2017 nov; 103(21); 1696-1703.

8. Andrew B. Goldstone, Peter Chiu, Michael Baiocchi: Mechanical or Biologic Prostheses for Aortic-Valve and MitralValve Replacement. n engl j med 377;19 nejm.org November 9, 2017

9. André Luiz Lisboa Cordeiro, Thiago Araújo de Melo: Inspiratory Muscle Training and Functional Capacity in Patients Undergoing Cardiac Surgery. Braz J Cardiovasc Surg 2016;31(2):140-4

10. Bender J: Heart valve disease. In: Zaret BL, Moser M, Cohen LS, eds. Yale University School of Medicine Heart Book. New York NY: Harper Collins; 2002: chap 13.

11. Bernie M Bisset, I Anne Leditschke: Protocol: Inspiratory muscle training for promoting recovery and outcomes in ventilated patients,(BMJ,2012;2:e000813. Doi.10.1136/bmjopen-2012-000813).

12. Bissett B, Leditschke IA, Neeman T, Boots R, Paratz J. Weaned but weary: one third of adult intensive care patients mechanically ventilated for 7 days or more have impaired inspiratory muscle endurance after successful weaning. Heart Lung. 2015;44(1):15-20.

13. Brasher PA, McClelland KH, Denehy L and Story I: Does removal of deep breathing exercises from a physiotherapy program including pre-operative education and early mobilisation after cardiac surgery alter patient outcomes, (Australian Journal of Physiotherapy 49: 165-173).
14. Carlos Gomez-Dumn, George J. Red, Jr: Indications for Heart Valve Replacement by Age Group. Kluwer Academic Publishers, Norwell, MA (1988)

15. Constantina Lomi and Elisabeth Westerdahl: Physical Therapy Treatment after Cardiac Surgery : A National Survey of Practice in Greece. J Clin Exp Cardiolog 2013, S7 DOI: 10.4172/21559880.S7-004 